\title{
Analysis of the effects of the Weider Pyramidal Technique for the development of muscular hypertrophy
}

\section{Análisis de los efectos de la Técnica Piramidal Weider para el desarrollo de la hipertrofia muscular}

Edgar Daniel Calderón Jaramillo. ${ }^{1}$, Frank Ruales Mosquera. ${ }^{2}$, Cristian Patricio Estrella Patarón. ${ }^{3}$ \& William Ricardo Caiza Núñez. ${ }^{4}$

\begin{abstract}
.
DOI: https://doi.org/10.33262/concienciadigital.v3i3.1.1393

The present research has as object of study, the theory and methodology in training and its field of action is based, within the strength training. In order to deepen the study of this capacity, the scope of exploration was delimited from the following objective. To analyze the effects of the Weider pyramidal technique on the muscular hypertrophy of the athletes of the Crows Gym in the city of Ambato. To guarantee the use of investigative sequences, the following actions were carried out: i) the scientific methodological sources that support the study of muscle hypotrophy and the weider pyramidal technique were examined and ii) anthropometric measurements were made before and after the application of a session of training. The scientific research approach was the quantitative product to which mathematical processing was used to assess the results of the use of the studied pyramidal technique and its possible influence on the performance of muscle hypertrophy. The fundamental methods used were synthetic, analytical, deductive inductive and measurement. The main results are a notable improvement in muscle hypertrophy performance from the application of the aforementioned technique and athletes at the time of their training must use technical varieties, each of the techniques in order to achieve the proposed objectives.
\end{abstract}

\footnotetext{
${ }^{1}$ Universidad Técnica de Ambato, Carrera de Cultura Física, Facultad de Ciencias de la Educación, Ecuador. edaniel2211@gmail.com, https://orcid.org/0000-0001-6554-2204

${ }^{2}$ Federación Deportiva Nacional del Ecuador, Departamento Técnico, Ecuador. fruales@ fedenador.org.ec https://orcid.org/0000-0002-6613-4373

${ }^{3}$ Universidad Técnica de Ambato, Carrera de Cultura Física, Facultad de Ciencias de la Educación, Ecuador. christiamestrella@hotmail.com, https://orcid.org/0000-0002-9684-3955

${ }^{4}$ Universidad Técnica de Ambato, Carrera de Cultura Física, Facultad de Ciencias de la Educación, Ecuador. ricardo_nuez@yahoo.es, https://orcid.org/0000-0003-2785-5589
} 
Keywords: Weider pyramidal technique, hypertrophic muscle, anthropometric measurements. Strength development.

\section{Resumen.}

La presente investigación tiene como objeto de estudio, la teoría y metodología del entrenamiento del fitness y se fundamenta su campo de acción, dentro del entrenamiento de la fuerza. Con el objetivo de profundizar en el estudio de esta capacidad se delimitaron los alcances de la exploración a partir del siguiente objetivo. Analizar los efectos de la técnica piramidal Weider en la hipertrofia muscular de los deportistas del Crows Gym de la ciudad de Ambato. Para garantizar su secuencia investigativa, se cumplieron con las siguientes acciones: i) se examinaron las fuentes científicas metodológicas que fundamentan el estudio de la hipertrofia muscular y la técnica piramidal weider y ii) se realizaron mediciones antropométricas antes y después de la aplicación de un programa de entrenamiento. El enfoque científico investigativo fue cuantitativo producto a que se utilizó un procesamiento matemático para valorar los resultados de la utilización de la técnica piramidal estudiada y su posible influencia en el desempeño de la hipertrofia muscular. Los métodos fundamentales utilizados fueron, analítico sintético, inductivo deductivo y la medición. Se obtienen como principales resultados una notable mejoría de las prestaciones de hipertrofia muscular a partir de la aplicación de la técnica antes mencionada y que los deportistas al momento de su entrenamiento deben utilizar variedades técnicas con el propósito lograr los objetivos propuestos.

Palabras Claves: Técnica piramidal weider, hipertrófica muscular, mediciones antropométricas. Desarrollo de la fuerza.

\section{Introducción.}

Las crecientes investigaciones acerca de los diferentes métodos para el desarrollo de la fuerza y dentro de esta, el aumento de la hipertrofia muscular es uno de los temas de hoy día llama la atención a expertos de todo el mundo. Para indagar sobre el tema en cuestión se realizó un profundo estudio en las bases de datos de la web of cience, scopus, cielo y latindex. Como resultado de esta revisión podemos afirmar que: En estos momentos existen diferentes criterios, ideas, así como recomendaciones, respaldadas por el posicionamiento del American College of Sports Medicine sobre el desarrollo de la hipertrofia muscular. Estos plantean que existen muchas variantes de intensidad del entrenamiento de esta dirección pero recomiendan que para incrementar la fuerza máxima o el tamaño muscular debe superar el $70 \%$ con 1 repetición máxima (RM)(ENTRO ANDALUZ MEDICINA DEL DEPORTE Volumen et al., 2011).

Otro estudio de significativa valía fue El método weider en la hipertrofia muscular de los jóvenes de 18 a 22 años del gimnasio elite de la ciudad de Ambato, donde se concluye que el $76 \%$ de los jóvenes practicantes que fueron sometidos al estudio, tienen conocimiento y utilizan el método Weider para su entrenamiento y desarrollo de masa muscular y fuerza, ya que este les permite un descanso adecuado a los músculos para una educada recuperación muscular. Los deportistas del Gimnasio Elite llevan una rutina de entrenamiento determinada en elevar las 
cargas de trabajo acrecentando su fuerza y estimulando la adecuada hipertrofia muscular durante todas sus sesiones de trabajo (Proaño, 2018, pág. 98).

Uno de los criterios de obligatoria consulta fue el entrenamiento de la fuerza y resistencia en hipoxia: efectos en la hipertrofia muscular (Fernández, Díaz, \& Caballero, 2019). Este tema brinda un apartado de relevancia para esta investigación ya que la ciudad de Ambato cuenta con una altura promedio de 2800 metros sobre el nivel del mar y aunque no podemos aseverar que las condiciones de hipoxia marcadamente elevadas, si resulta oportuno revisar la investigaciones acerca de este caso, donde se concluye que la sinergia del entrenamiento y las condiciones de hipoxia producen una mayor adaptación y cambios favorables en la fisiología muscular de los tejidos musculares y por ende trae consigo cambios fenotípicos muy favorables en el desarrollo de la hipertrofia sobre todo cuando se constatan condiciones reales de hipoxia. Considerando la dosis de hipoxia, la duración de las sesiones, la intensidad del ejercicio, la frecuencia semanal, las semanas de tratamiento y el número de sesiones. Además, deben contemplarse los aspectos dietéticos y nutricionales, así como la administración de los nutrientes necesarios.

Una revisión bibliográfica sobre los sistemas de entrenamiento y los métodos convencionales con pesas y sus usos Rincón Galvis Daniel A. (2018) plantea que el hecho de que en diferentes espacios se sugieran nuevas tendencias para el proceso de entrenamiento de la hipertrofia con propuesta incluso novedosas, estas no han provocado que el entrenamiento convencional con pesas no deja de ser utilizado como fuente principal para general la hipertrofia. Por una parte, porque aún se encuentran gimnasios que ofrecen el servicio de entrenamiento con pesas, así como deportistas de alto rendimiento que realizan sus respectivos entrenamientos con estos sistemas, investigaciones que estudian los efectos del entrenamiento con pesas en distintos escenarios, ya sea para rehabilitación o para el desarrollo de capacidades físicas Y, por otro lado, porque los sistemas no convencionales, dentro de su rutina de entrenamiento o sesiones, implementan los métodos y sistemas de entrenamiento convencional como lo es el trabajo en circuito conocido como HIIT, el cual se demostró en la discusión viene de los años 40, elementos de halterofilia son implementados en el crossfit, el trabajo con el peso corporal continúa siendo un trabajo de auto carga. (Rincón Galvis Daniel A 2018) citado por (Edgar Daniel Calderón Jaramillo, 2019). Además, según Villalba \& Ocio Aitor (2015) estos proporcionan que diferentes grupos musculares con su mecánica de ejecución realicen los distintos ejercicios que forman parte del sistema o método de entrenamiento, la cual esta se concentra en la hipertrofia y definición muscular.

Pero por otra parte Cometti, (2005) citado por (Edgar Daniel Calderón Jaramillo, 2019). establece que en la hipertrofia es la causa de la ganancia de fuerza muscular, el desarrollo de la fuerza depende de tres factores importantes los cuales son: estructurales, nervioso y capacidad de contracción del músculo. La hipertrofia es un factor estructural depende de la sección del músculo y se dan por aumento de miofibrillas, un desarrollo de las envolturas del músculo, aumento de vascularización 
En este sentido, no podemos olvidar el entrenamiento funcional, como concepto aparte del convencional, está relacionado a este por los principios y normas que hacen parte del trabajo de la fuerza y además que garantizan el desarrollo de las zona (Chango-Sigüenza \& MontoroBombú, 2018) citado por (Edgar Daniel Calderón Jaramillo, 2019); es este caso, para objetivos estructurales. Por lo tanto, el entrenamiento convencional, actualmente es utilizado ya sea en establecimientos propios del entrenamiento con pesas, o en la aplicación de las modalidades del entrenamiento funcional. Además de que es implementado en investigaciones o el entrenamiento de otras disciplinas deportivas (Rincón Galvis Daniel A. 2018).

\section{Metodología.}

La presente investigación tubo un enfoque cuantitativo, debido que se utilizaron procesos matemáticos para valorar los resultados obtenidos de la Técnica Piramidal Weider y su incidencia en la hipertrofia muscular. (Edgar Daniel Calderón Jaramillo, 2019)

En la investigación se utilizó la información sobre las dos variables que se utilizó durante este proyecto, investigaciones sobre la técnica piramidal Weider que pertenece a los principios de entrenamiento en el área de la musculación, y en cuanto a la otra variable que es la hipertrofia muscular. (Edgar Daniel Calderón Jaramillo, 2019)

Según Hernández (1998) estableció que el diseño de preprueba - post prueba con un solo grupo: A un grupo se le aplica una prueba previa al estímulo o tratamiento experimental; después se le administra un instrumento de intervención o tratamiento y finalmente se le aplica una prueba posterior al tratamiento, entonces:

La base de la investigación fue la toma de medidas antropométricas de los 30 deportistas del gimnasio Crows Gym, cuyas edades fueron entre 19 y 35 años, para establecer un punto de partida en cuanto a los niveles de masa muscular poseían, previo a la aplicación de una rutina de entrenamiento basado en la técnica piramidal Weider. (Edgar Daniel Calderón Jaramillo, 2020)

Tras el proceso de recolección de datos finalizado, se analizó la variable independiente que es la técnica piramidal Weider, siendo esta un principio para determinar en caso de que tuviera un efecto o no en la variable dependiente que es la hipertrofia muscular.

El pre-experimento que se ejecutó en distintas fases:

Fase 1; Pre-Valoración Antropométrica: Se realizo una pre-evaluación de medidas antropométricas (perímetros) de los grupos musculares como son Bíceps en Relajado y en Contracción, Pectoral (Pecho), Muslo y Gemelos (Pantorrilla), previa a la ejecución de la rutina de entrenamiento para establecer los perímetros con los cuales los deportistas iniciaron.

Fase 2; Aplicación de rutina de entrenamiento basado en la Técnica Piramidal: Se aplico a los deportistas la rutina de entrenamiento basado en la técnica piramidal Weider, la cual tubo la duración de un periodo de tiempo de 7 semanas.

Fase 3; Post-Valoración Antropométrica: Tras finalizar el periodo de ejecución de la rutina de entrenamiento, se procedio a realizar una post-evaluación de las medidas antropométricas 
(perímetros) de los grupos musculares como son Bíceps en Relajado y en Contracción, Pectoral (Pecho), Muslo y Gemelos (Pantorrilla), para descubrir si se produjo un cambio o no en las medidas de la pre-valoración antropométrica

Se determino el incremento porcentual mediante el uso de la fórmula de incremento porcentual (Montoro Bombú et al., 2019) modificada por el autor.

Instrumento de evaluación: Pre y Post Mediciones antropométricas "Perímetros" Normas Internacionales para la Valoración Antropométrica de los siguientes grupos musculares, se utilizará el manual establecido por la Sociedad internacional para el avance de la cineantropometría "ISAK” (2005). Citado por Daniel Edgar Calderón Jaramillo (2020)

- Bíceps en Relajado y en Contracción

- Pectoral (Pecho)

- Muslo

- Gemelos (Pantorrilla)

Instrumento de intervención: Rutina de Entrenamiento basado en la Técnica Piramidal Weider

Población: 100 Deportistas varones

Muestra: 30 Deportistas Varones en las edades de 19 a 35 años

El análisis estadístico se realizó mediante el paquete estadístico SPSS (T DE STUDENTS) un análisis de resultados obtenidos mediante la rutina de entrenamiento basado en la técnica piramidal Weider.

\section{Resultados.}

Gráfico 1. Pre-Post Valoración Bíceps Relajado

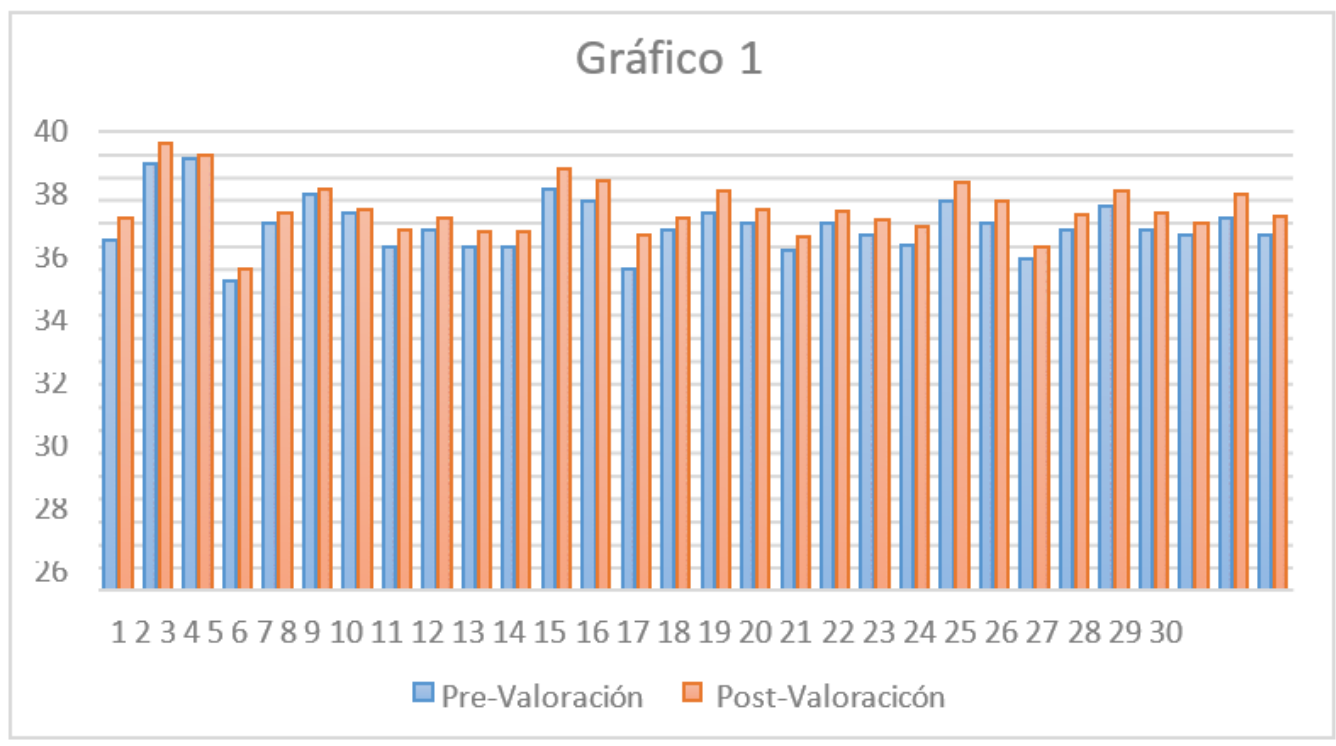

Fuente: Elaboración propia. 


\section{Análisis.}

De la totalidad de la muestra de 30 deportistas, después de la aplicación de la rutina de entrenamiento y en el análisis entre la pre-valoración y la post-valoración se determinó que todos los deportistas obtuvieron un aumento en su hipertrofia muscular, cabe destacar que el $50 \%$ de los deportistas, superan la media del aumento de la hipertrofia que es de $1.4 \mathrm{~cm}$ en ese grupo muscular, mientras que el otro $50 \%$ de deportistas tuvieron un aumento de su hipertrofia sin superar la media, El grupo muscular del bíceps en estado de relajación se observa con facilidad el incremento de masa muscular, la totalidad de los deportistas han obtenido un aumento en distintas medidas en su hipertrofia muscular después de la aplicación de la rutina de entrenamiento.

Gráfico 2. Bíceps Contraído

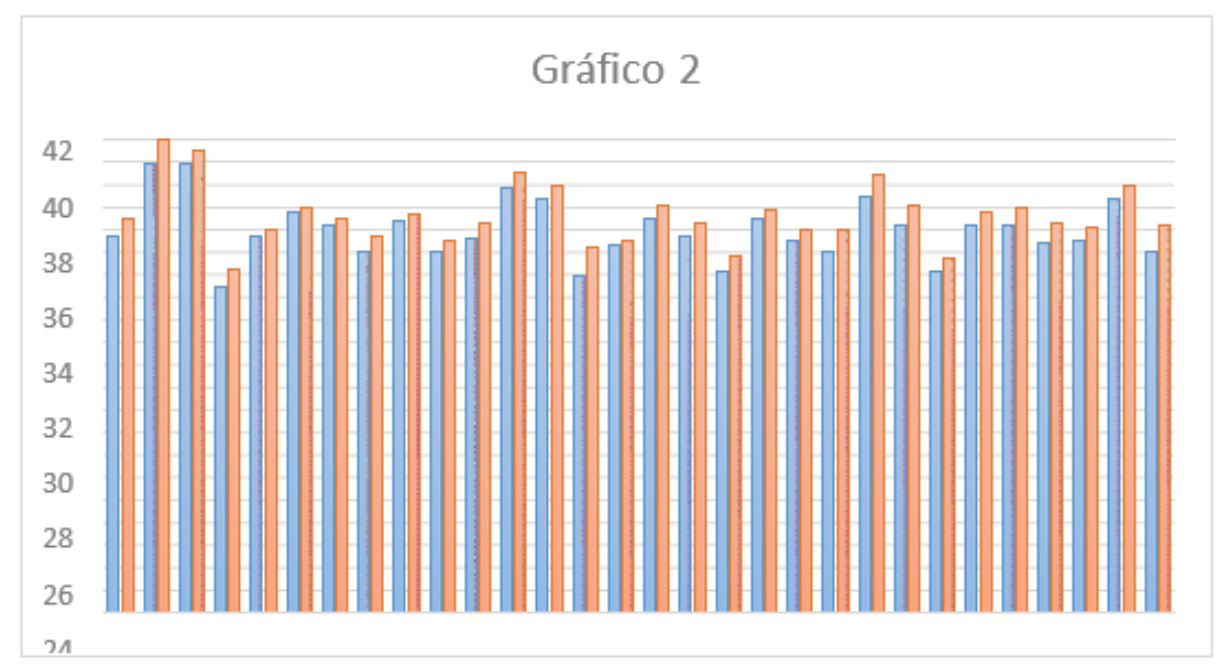

Fuente: Elaboración propia.

\section{Análisis.}

De la totalidad de la muestra de 30 deportistas posterior a la aplicación de la rutina de entrenamiento y en el análisis entre la pre-valoración y la post-valoración se determinó que todos los deportistas obtuvieron un aumento en su hipertrofia muscular, cabe diferenciar que el $43 \%$ de los deportistas, superan la media del aumento de la hipertrofia muscular que es de 1.3 $\mathrm{cm}$ en ese grupo muscular, mientras que el $6 \%$ de deportistas tuvieron un aumento de su hipertrofia hasta llegar a la media, mientras el 50\% de la muestra si obtuvieron un aumento en su hipertrofia pero no lograron superar la media en ese grupo muscular, Después de la aplicación de la rutina de entrenamiento en el grupo muscular del bíceps en estado de contracción se observa que todos los deportistas aumentaron la masa muscular, la totalidad de los deportistas han obtenido un aumento en distintas medidas en sus fibras musculares. Esto provocó una gran motivación y pudimos constatar lo planteado por disímiles autores coinciden en afirmar que la motivación determina la dirección, intensidad y el sentido del comportamiento humano. (Inelvis Romero Pileta et al., 2019) citado por Edgar Daniel Calderón Jaramillo (2020) 
Gráfico 3. Pre-Post Valoración Pectoral

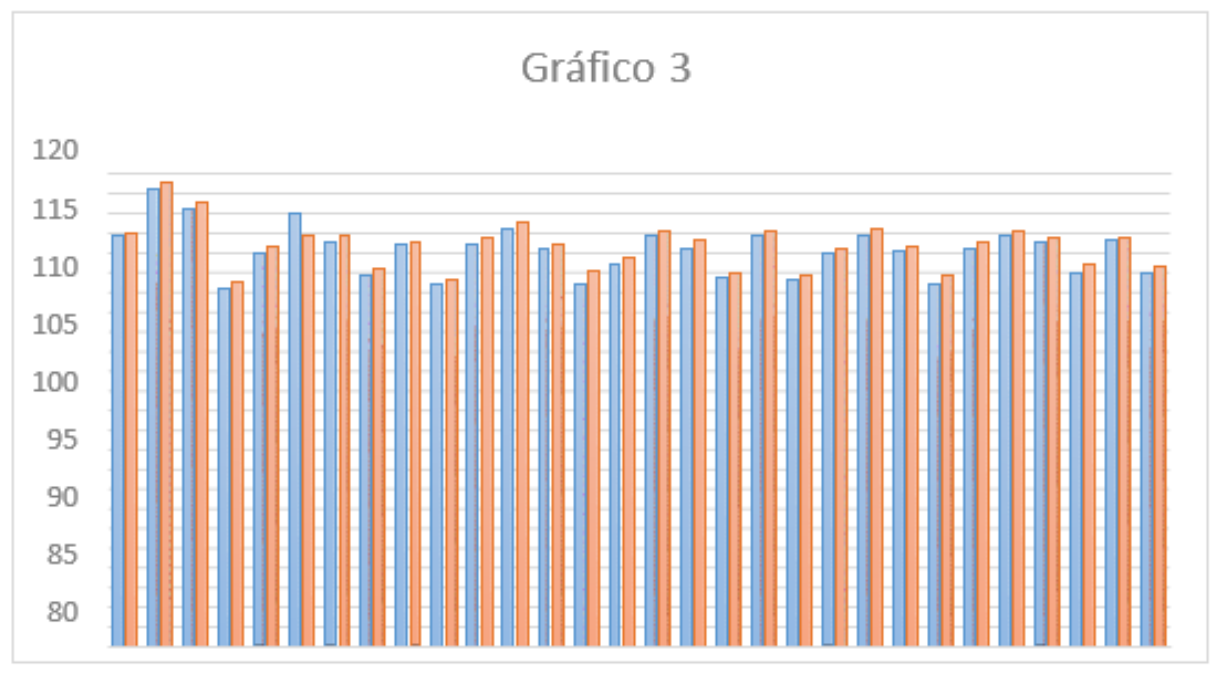

Fuente: Elaboración propia.

\section{Análisis.}

De la totalidad de la muestra de 30 deportistas, el análisis entre la pre-valoración y la postvaloración, determinó un $97 \%$ obtuvieron un aumento en su hipertrofia muscular, de los cuales el $63 \%$ de deportistas superan la media del aumento de la hipertrofia que es de $1.2 \mathrm{~cm}$ en ese grupo muscular, mientras que $7 \%$ de deportistas tuvieron un aumento de su hipertrofia que se mantuvo en la media, mientras que $23 \%$ de deportistas si obtuvieron un aumento en su hipertrofia pero no lograron superar la media, el caso específico de un $4 \%$ de deportistas redujo su hipertrofia muscular. Todos los deportistas obtuvieron un aumento en su hipertrofia muscular excepto un deportista que redujo su masa muscular.

Gráfico 4. Pre-Post Valoración Muslo

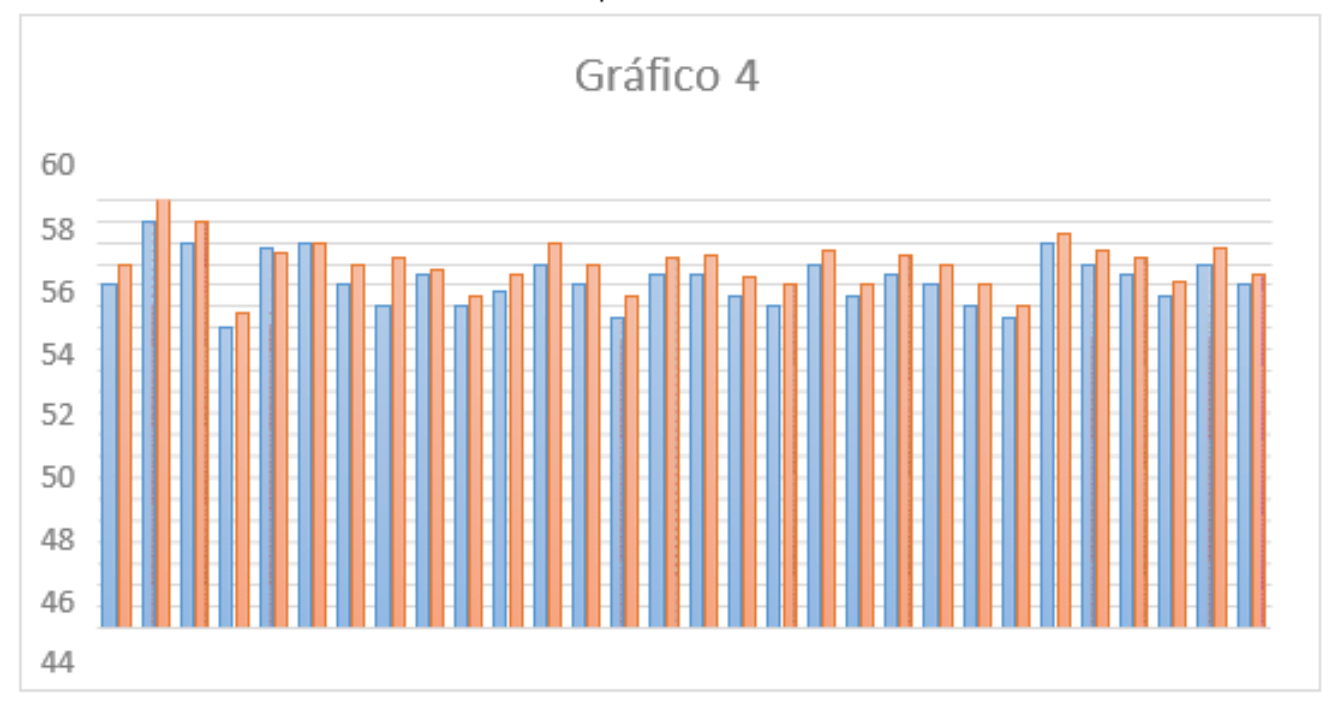

Fuente: Elaboración propia. 


\section{Análisis.}

De la totalidad de la muestra de 30 deportistas el análisis entre la pre-valoración y la postvaloración se determinó que un $97 \%$ obtuvieron un aumento en su hipertrofia muscular, de los cuales $50 \%$ de deportistas que representan superan la media del aumento de la hipertrofia que es de $1.5 \mathrm{~cm}$ en ese grupo muscular, mientras que $7 \%$ deportista tuvieron un aumento de su hipertrofia que se mantuvo en la media, mientras que $37 \%$ de deportistas si obtuvieron un aumento en su hipertrofia pero no lograron superar la media, los casos especiales como son de $3 \%$ de deportistas que mantuvo su hipertrofia y que otro $3 \%$ redujo su hipertrofia muscular

Gráfico 5. Pre-Post Valoración Pantorrilla

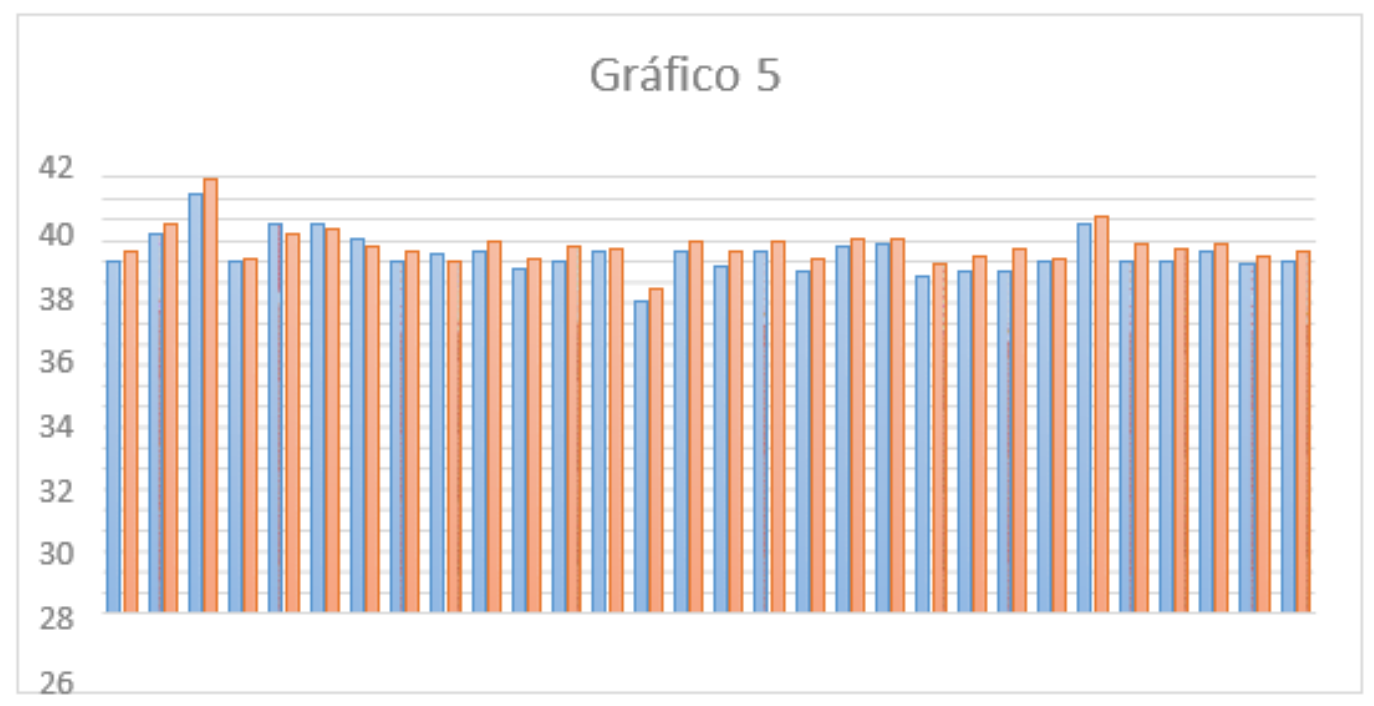

Fuente: Elaboración propia.

\section{Análisis.}

De la totalidad de la muestra de 30 deportistas el análisis entre la pre-valoración y la postvaloración se determinó que 26 deportistas que representan un $87 \%$ obtuvieron un aumento en su hipertrofia muscular, de los cuales el $57 \%$ deportistas superan la media del aumento de la hipertrofia que es de $0.8 \mathrm{~cm}$ en ese grupo muscular, mientras que 7\% deportista tuvieron un aumento de su hipertrofia que se mantuvo en la media, mientras que $23 \%$ de deportistas si obtuvieron un aumento en su hipertrofia pero no lograron superar la media, los casos especiales como son un $13 \%$ del total se produjo una disminución de la hipertrofia muscular.

\section{Discusión.}

La hipertrofia muscular es nombre entregado a la manifestación del crecimiento en el tamaño de las células musculares, según Cometti (2005) establece que en la hipertrofia es la causa de la ganancia de fuerza muscular, el desarrollo de la fuerza depende de tres factores importantes los cuales son: estructurales, nervioso y capacidad de contracción del músculo. La hipertrofia es un factor estructural depende de la sección transversal del músculo y se dan por aumento de miofibrillas, un desarrollo de las envolturas del músculo, aumento de vascularización, lo cual supone un aumento de tamaño de las fibras musculares y por lo tanto del músculo (pág. 30). 
Entonces es el crecimiento de las células musculares sin que exista una división celular (Hiperplasia), entonces se establece que el músculo estimulado a este proceso ofrece por igual una mejor respuesta a la carga.

La hipertrofia muscular es el incremento del tamaño de las fibras musculares en dimensiones transversales (perímetro), la hipertrofia muscular se relaciona en conjunto con la fuerza, estableciendo que al poseer más masa muscular tiene mayor fuerza. La técnica piramidal Weider permite la obtención de los mecanismos de la hipertrofia muscular que son la tensión mecánica, estrés metabólico y daño muscular, los cuales desencadenan una serie de reacciones bioquímicas que culminan en el proceso de hipertrofia muscular, la técnica piramidal Weider permite el trabajo con intensidad y volumen de carga, ya sean intensos o moderados y esto permite un crecimiento en las fibras musculares. Pero estos tienen que ser constantemente controlados para que sus efectos se vean reflejados en la práctica (Montoro Bombú et al., 2018)

El Sistema Weider existe desde hace más de 60 años y ha ido cambiando y actualizándose, según se van haciendo nuevos descubrimientos. No solo es un sistema de entrenamiento, sino que también permite guiar a diseñar una rutina de entrenamiento adaptado a cada individuo basándose en factores de cada deportista.

En un estudio realizado por Proaño (2018) el 80\% de los integrantes del gimnasio Elite están de acuerdo que utilizando el método de entrenamiento Weider se logra elevar más cargas de trabajo y el 20\% considera que no. Y el $46 \%$ de los integrantes del gimnasio Elite conocen los principios que emplea este método y el $54 \%$ no las conocen.

\section{Conclusiones.}

- La evaluación de los parámetros antropométricos logró determinar que cada deportista posee diferentes medidas antropométricas en cada grupo muscular previo a la aplicación de la rutina de entrenamiento, las diferentes evaluaciones antropométricas que resultan en distintas medidas de cada individuo son factores establecidos por distintos componentes relacionados al cuerpo humanos como son el de la anatomía y fisionomía humana.

- Se aplicó de manera correcta una rutina de entrenamiento basado en la técnica piramidal Weider con una muestra de 30 deportistas y el tiempo que duro la aplicación de rutina fue de duración de 7 semanas.

- Tras la aplicación de una rutina de entrenamiento basado en la técnica piramidal Weider se logró determina las diferencias existentes entre las medias de cada grupo muscular; la diferencia de media del bíceps en estado de relajación es de $1.4 \mathrm{~cm}$, del bíceps en contracción es de $1.3 \mathrm{~cm}$, del musculo pectoral es $1.4 \mathrm{~cm}$, del muslo es de $1.6 \mathrm{~cm}$, de la pantorrilla es de $0.8 \mathrm{~cm}$, entonces se comprende que si ha existido un aumento en la hipertrofia muscular de cada grupo muscular. 


\section{Referencias Bibliográficas.}

Chango-Sigüenza, M., \& Montoro-Bombú, R. (2018). Artículo Original El desarrollo de las zonas funcionales en la marcha deportiva y las carreras de fondo Development of functional areas in middle-fund distance run and sport march. Revista Acción, 14, 16083792. http://accion.uccfd.cu

Cometti, G. (2005). Los métodos modernos de la musculación. En g. Cometti.

Paidotribo.

Daniel Edgar Calderón Jaramillo. (2019). UNIVERSIDAD TECNICA DE AMBATO FACULTAD DE CIENCIAS HUMANAS Y DE LA EDUCACION CARRERA DE CULTURA FÍSICA MODALIDAD PRESENCIAL.

ENTRO ANDALUZ MEDICINA DEL DEPORTE Volumen, C. DE, Martín-Hernández, J., \& Marín Herrero, P. A. (2011). Medicina del Deporte Revisión de los procesos de hipertrofia muscular inducida por el entrenamiento de fuerza oclusivo. Rev Andal Med Deporte, 4(4),

152-157. http://http//zl.elsevier.esel05/09/2013.Copiaparausopersonal,seprohíbelatransmisiónde estedocumentoporcualquiermediooformato.

Fernández, D., díaz, J., \& Caballero, A. (2019). Entrenamiento de fuerza y resistencia en hipoxia: efecto en la hipertrofia muscular. Biomédica, vol. 39, núm. 1, 2019, 39(1), 9.

Inelvis Romero Pileta, Hirbins Manuel Dopico Pérez, Idalmis Fernández Téllez, Raynier Montoro Bombú, Enrique Chávez Cevallos, \& Wilson Teodoro Contreras Calle. (2019, junio). Análisis integral de la motivación en boxeadores. http://scielo.sld.cu/scielo.php?script=sci_arttext\&pid=S0864- 03002019000200056

Montoro Bombú, R., Hernández Toro, V., Ortiz Ortiz, P., \& Castro Acosta, W. (2019). Contribuciones al control físico-pedagógico del rendimiento deportivo de los corredores de $400 \quad \mathrm{~m}$ planos. Ciencia Digital, 3(2.5), 32-45. https://doi.org/10.33262/cienciadigital.v3i2.5.528

Proaño, J. Y. (2018). El método weider en la hipertrofia muscular de los culturistas jovenes de 18 a 22 años del gimnasio elite”. Págs. 98-99

Rincón, G. D. (2018). Revisión bibliográfica los sistemas y métodos del entrenamiento convencional con pesas y su uso. 43.

Sampieri, R. H. (2013). Metodologia de la investigación (sexta ed.). Mcgraw hill.

Weider, J. (1988). Joe weider’s bodibuilnding sistem. En J. Weider. 
PARA CITAR EL ARTÍCULO INDEXADO.

Calderón Jaramillo, E. D., Ruales Mosquera, F., Estrella Patarón, C. P., \& Caiza Núñez, W. R. (2020). Analysis of the effects of the Weider Pyramidal Technique for the development of $\begin{array}{llll}\text { muscular } \quad \text { hypertrophy. } & \text { ConcienciaDigital, } & 3(3.1), & \text { 257-267. }\end{array}$ https://doi.org/10.33262/concienciadigital.v3i3.1.1393

\section{LCiencia}

El artículo que se publica es de exclusiva responsabilidad de los autores y no necesariamente reflejan el pensamiento de la Revista Conciencia Digital.

El artículo queda en propiedad de la revista y, por tanto, su publicación parcial y/o total en otro medio tiene que ser autorizado por el director de la Revista Conciencia Digital.
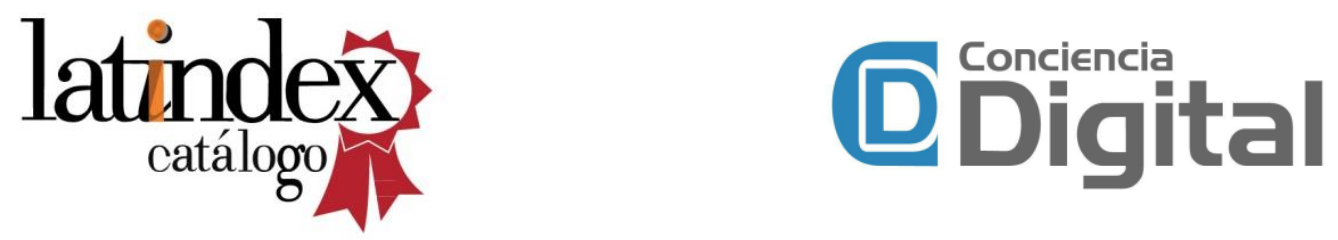